\title{
Tunable, Grating-Assisted Single-Ring Laser Mirrors
}

\author{
Carmen Vázquez ${ }^{1}$, and Otto Schwelb ${ }^{2}$ \\ ${ }^{1}$ Departamento Tecnología Electrónica, Universidad Carlos III, Leganés 28911, Madrid, Spain \\ ${ }^{2}$ Electrical and Computer Engineering, Concordia University, Montréal, QC, Canada, H3G 1M8
}

\begin{abstract}
Two configurations based on Bragg grating assisted single-ring microresonators are analyzed and numerically simulated. Device characteristics such as bandwidth, sensitivity to loss, effect of external coupling and tunability are simulated. The relationship between the resonator mode number and the grating parameters and the selection rules for appropriate mode numbers are discussed. Parametrized reflection characteristics illustrate the analytical results.

Index Terms - Gratings, integrated optics, mirrors, optical resonators, optical waveguide components.
\end{abstract}

\section{INTRODUCTION}

Microring resonator based optical mirrors and band-limited reflectors have been the subject of intense investigations in recent years, primarily due to the advancement in fabrication technology and miniaturization of integrated devices [1]-[6]. Historically, the first fiber optic mirror, consisting of a feedback-assisted coupler, has been described by Mortimore [7]. The functioning of this mirror, where the output ports of a fused coupler are connected by a length of optical fiber, was based on the $90^{\circ}$ phase difference between the coupled and direct paths of the coupler.

This paper presents analytical and simulated results on the performance of two closely related narrow-band laser mirror configurations. Both consist of a microring resonator to which, through a coupler, are attached two identical Bragg gratings. The first configuration, illustrated schematically in Fig. 1a) has been discussed in a different context in [8]-[11] while the second configuration, shown in Fig. 1b), was briefly treated in [12]. Although they share similarities, their response and fabrication differs in respects that could determine the choice of application. For example, Fig. 1a) indicates a coupler geometry connecting the resonator to the Bragg waveguides that requires bends of very small radii which might necessitate the use of vertical coupling technology. Other aspects differentiating the configurations are tunability, sensitivity to loss, group delay in reflection, bandwidth, and the most convenient positioning of (thermo-optic) tuning pads.

In what follows we shall discuss the analytical aspects of treating these devices, including a circuit reduction scheme that simplifies the arithmetic, and an analysis of the resonance spectrum of the devices disconnected from the external circuitry, followed by computed results covering reflected intensity responses, mode number optimization, return loss and tuning sensitivity.
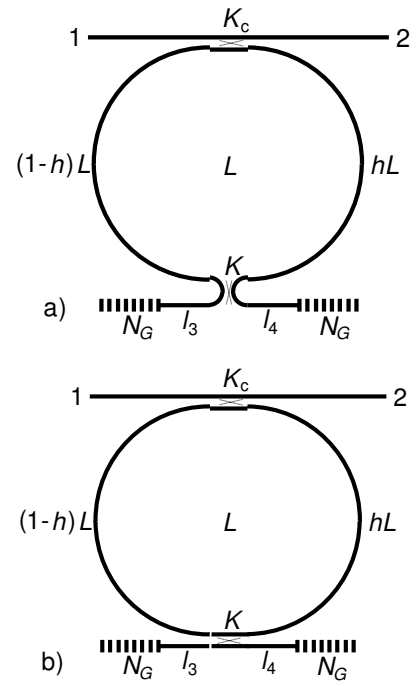

Fig. 1. Two grating-assisted microring resonator laser mirrors. The ring perimeter is $L$ and $0<h<1$ locates the coupler $K$ on the ring.

\section{ANALYSIS}

Referring to Fig. 1, $K$ represents the power coupling coefficient of the coupler while the location of $K$ within the ring, determined by $h(0<h<1)$, is arbitrary. All four transmission line sections, namely $h L,(1-h) L, l_{3}$ and $l_{4}$, are characterized by the same effective refractive index $n_{\text {eff }}$ and the same amplitude attenuation coefficient $\alpha$, although this restriction can easily be relaxed. We also admit localized or distributed gain in the ring [13]-[15] and thermo-optic length or refractive index adjustment of $L, l_{3}, l_{4}$ and $n_{\text {eff. }}$. All lengths are given in terms of mode numbers, for example, $N=L n_{\text {eff }} / \lambda_{0}$, where $\lambda_{0}$ is the design wavelength. Another important parameter is the round trip time delay $\tau_{0}=N / f_{0}$, where $f_{0}$ is the design frequency. The gratings are identical, quarter wavelength shallow gratings, meaning that the relative index difference $\left(n_{b}-n_{a}\right) / n_{a} \quad 1$, and $N_{G}$ is the number of periods. To ensure balanced operation normally $K \cong 0.5$, whereas $K_{c}$ is small to prevent loading and to preserve the resonance spectrum of the grating-assisted ring.

Figs. 1a) and 1b) can be simplified by combining the reflectors and the coupler $K$ into a single two-port and embedding this two-port into the ring, as shown in Fig. 2. The 
$2 \times 2$ scattering matrix $\boldsymbol{S}_{K}$ can be calculated from first principles and is found to be

$$
S_{k}=\left[\begin{array}{cc}
c^{2} \Gamma_{3}-s^{2} \Gamma_{4} & j s c\left(\Gamma_{3}+\Gamma_{4}\right) \\
j s c\left(\Gamma_{3}+\Gamma_{4}\right) & c^{2} \Gamma_{4}-s^{2} \Gamma_{3}
\end{array}\right]
$$

in the case of Fig. 1a) and

$$
\mathrm{S}_{\mathrm{K}}=\frac{1}{1-\mathrm{c}^{2} \Gamma_{3} \Gamma_{4}}\left[\begin{array}{cc}
-\mathrm{s}^{2} \Gamma_{4} & \mathrm{c}\left(1-\Gamma_{3} \Gamma_{4}\right) \\
\mathrm{c}\left(1-\Gamma_{3} \Gamma_{4}\right) & -\mathrm{s}^{2} \Gamma_{3}
\end{array}\right]
$$

for Fig. 1b). Here we used $c=\sqrt{1-K}$ and $s=\sqrt{K}$, and

$$
\Gamma_{i}=\frac{-\mathrm{j} \kappa^{*} \operatorname{sh}\left(\gamma_{\mathrm{G}} \mathrm{N}_{\mathrm{G}, \mathrm{i}} \Lambda\right)}{\gamma_{\mathrm{G}} \operatorname{ch}\left(\gamma_{\mathrm{G}} \mathrm{N}_{\mathrm{G}, \mathrm{i}} \Lambda\right)+(\alpha+\mathrm{j} \Delta \beta) \operatorname{sh}\left(\gamma_{\mathrm{G}} \mathrm{N}_{\mathrm{g}, \mathrm{i}} \Lambda\right)} \mathrm{e}^{\left[-2(\alpha+\mathrm{j} \beta)_{\mathrm{i}}\right]}
$$

are the complex reflection coefficients of arms 3 and 4, consisting of guide lengths $l_{3}$ and $l_{4}$, respectively, terminated by their corresponding gratings of lengths $N_{G, i} \Lambda$, where $\Lambda$ is the periodicity, $N_{G}$ the number of unit cells, $\Delta \beta=\beta-\pi / \Lambda$ the detuning, $\gamma_{\mathrm{G}}=\sqrt{|\kappa|^{2}+(\alpha+\mathrm{j} \Delta \beta)^{2}}$ and $\kappa$ is the coefficient that measures the coupling between forward and backward traveling waves in the grating [16]. We evaluated the spectral response of the devices also using flat dielectric mirrors in place of the Bragg gratings, but these results are not reported here, because the practically wavelength independent reflection coefficient of the dielectric mirror invalidates the main reason for using gratings, namely to tailor the reflection spectrum and to prevent its recurrence shifted by the free spectral range (FSR).

The main parameters that determine the performance of the composite mirror are the optical lengths $L n_{\text {eff }}, l_{3} n_{\text {eff }}, l_{4} n_{\text {eff }}$, the coupling coefficient to the bus waveguide $K_{\mathrm{c}}$, the magnitude and phase of the reflection coefficients $\Gamma_{I}$, and the attenuation coefficient of the waveguides $\alpha$. The computed characteristics include the reflected intensity and phase, the group delay in reflection, the return loss and the insertion loss, not all of which can be reported here due to space restrictions.

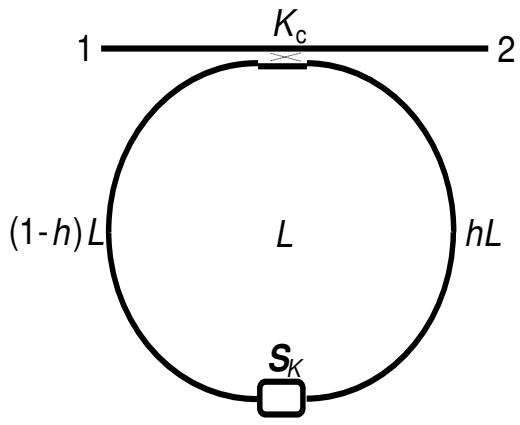

Fig. 2. A reduced schematic representation of the mirrors of Fig. 1. The reflectors at the end of arms $l_{3}$ and $l_{4}$, and the coupler $K$ are combined in the two-port $\boldsymbol{S}_{K}$ embedded in the ring.

\section{SPECTRAL CHARACTERISTICS}

Without the band limiting effect of the Bragg gratings the reflection characteristic of the composite mirror would display a periodic behavior with a $F S R$ determined by the ring perimeter according to $F S R=f_{0} n_{\text {eff }} / N n_{g}$, where $n_{g}$ is the group index. This is the type of reflection characteristic obtained when the gratings are replaced with constant reflection coefficient dielectric mirrors. The bandwidth of the grating, determined by $\kappa$, must be adjusted therefore to be significantly less than $2 \times F S R$. This is illustrated in Fig. 3, showing, for Fig. $1 \mathrm{a})$, the reflected intensity as a function of detuning for three mode numbers $N$. Observe that as $N$ increases and the FSR decreases the suppression in the wings deteriorates.

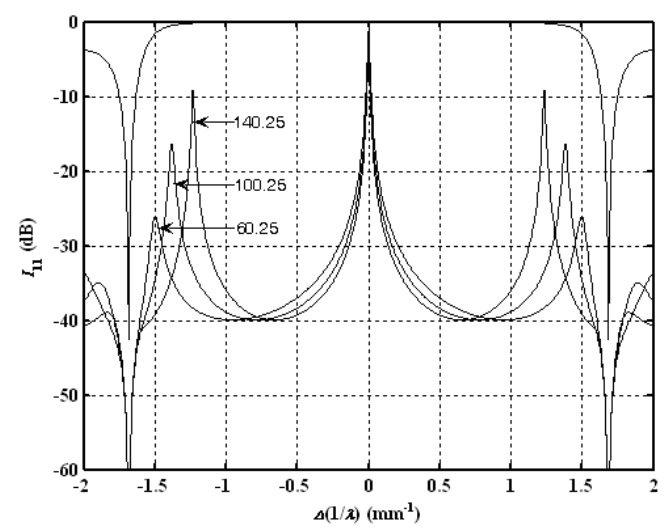

Fig. 3. Reflected intensity response of the mirror of Fig. 1a) for three mode numbers. Also shown is the return loss of the gratings. The parameters were: $N_{G}=600, n_{a}=1.5098, n_{b}=n_{\text {eff }}=1.5, K=0.5$, $K_{c}=0.02, \lambda_{0}=1.5791 \mu \mathrm{m}, N_{3}=10.25, N_{4}=10$ and $\alpha=0$. Multiply the abscissa by $3 \times 10^{2}$ to obtain the detuning in $\mathrm{GHz}$.

There are certain selection rules for the three mode numbers, $N, N_{3}$ and $N_{4}$ which determine the lengths $L, l_{3}$ and $l_{4}$, respectively, that one must obey to obtain a reflection characteristic centered at the design frequency $f_{0}$. These are due to the need for a constructive interference for the reflected signal at port 1 and take into account the reflection phase of $\Gamma_{3}$ and $\Gamma_{4}$, and the $\pi / 2$ phase shift between the coupled and direct arms of $K$. The selection rules are numerous and too multifarious to be listed or tabulated here, we note, however, that Figs. 1a) and 1b) have each their own advantages and disadvantages when it comes to tuning and sideband suppression.

As a comparison to Fig. 3, we present in Fig. 4 the reflection characteristics of the mirror device of Fig. 1b), obtained using the same network parameters. Although here the sidelobe suppression appears to be better, the overall performance might not point clearly in one direction.

To help determine the locations of the main and subsidiary reflection peaks that fall within the passbands of the gratings we remove the external bus waveguide by setting $K_{c}=0$, and solve the appropriate determinantal equation to find the resonances of the remaining free-standing resonator. The 
details of this procedure have recently been described elsewhere [17], here only the equivalent circuits of the resonators in question are shown and some of the spectra plotted.

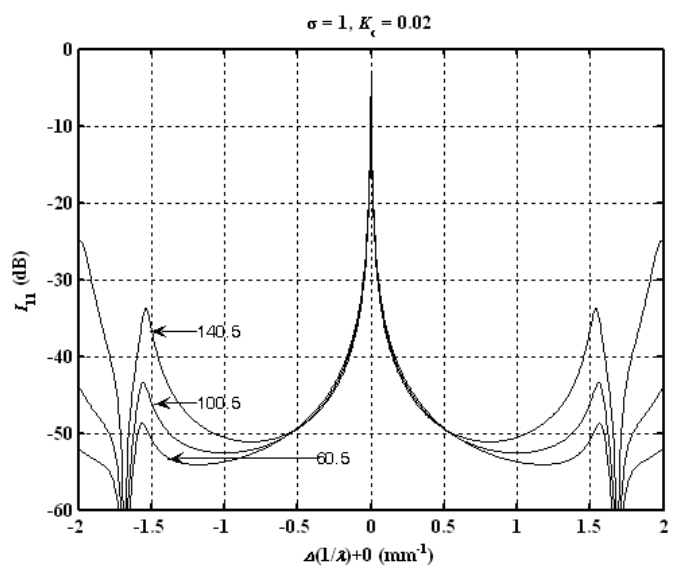

Fig. 4. Reflection response of the mirror of Fig. 1b), for three values of $N$. The remaining circuit parameters are identical to those given in the previous diagram, $\sigma=\exp (-\alpha L)$.

The equivalent circuits of Fig. 1, after setting $K_{c}=0$, are shown in Fig. 5a) and 5b), respectively. The only difference between them is the type of coupler connecting the ring with the gratings. In the schematics the solid and dotted lines represent the direct and coupled connections, respectively. If the required bending radius proves to be prohibitively small in Fig. 1a) and, as suggested in the Introduction, one must resort to vertical coupling then the connections in Fig. 5a) can be interchanged. The minima, or zeros in case of a lossless system, of the appropriate determinantal equation indicate the spectral locations of the resonances. These appear at the same wavelengths as the reflection peaks of the composite mirror, as long as $K_{c}$ is sufficiently small. This is usually the case attempting to prevent undue loading by the external circuitry. Figs. 6 and 7 plot the resonance spectra corresponding to Figs. 3 and 4, indicating a very close agreement between the location of the resonances and the peaks of the sidelobes.

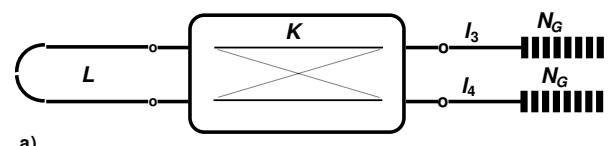

a)

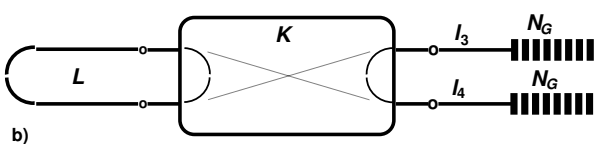

Fig. 5. Schematic diagrams of the free-standing resonators remaining after setting $K_{c}=0$ in Fig. 1a) and 1b), respectively. $L$ represents the microring. The direct and coupled connections of $K$ are the solid and dotted lines, respectively.

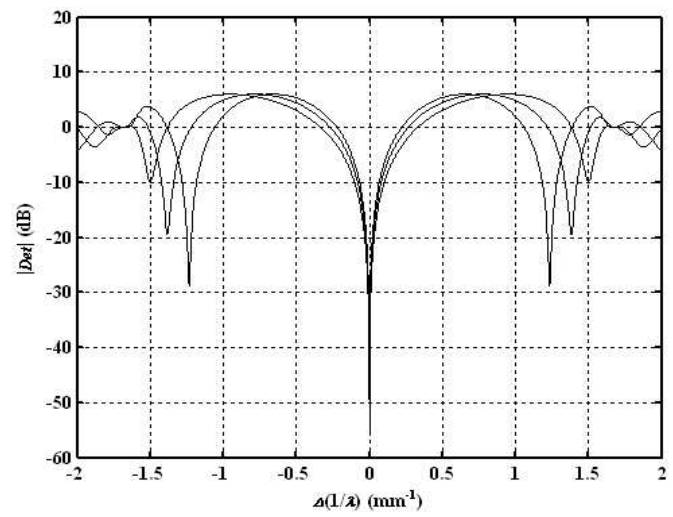

Fig. 6. Resonance spectrum of of the free-standing resonator obtained by setting $K_{c}=0$ in Fig. 1a). The minima locate the resonances. Loss is due only to finite grating length.

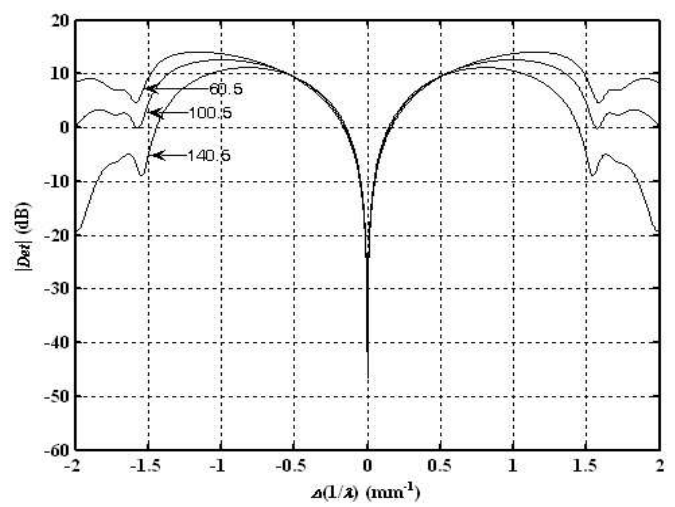

Fig. 7. Resonance spectrum of of the free-standing resonator obtained by setting $K_{c}=0$ in Fig. 1b). The minima locate the resonances. Loss is due only to finite grating length.

\section{RETURN LOSS, TUNABILITY AND BANDWIDTH}

Two loss contributions are considered, namely waveguide loss and grating loss. The former characterizes all waveguide sections, including the gratings, with a common attenuation coefficient $\alpha$, while the latter is due to leakage at the far end of the finite gratings. The couplers are assumed to be lossless, but our simulation program is equipped to take coupler loss into account. Ideally, the two loss contributions should be in approximate balance. Return loss $(R L)$ measures, in $\mathrm{dB}$, the maximum of $I_{11}$ at $f_{0}$ for a properly tuned mirror. We found that the mirror of Fig. 1a) had significantly smaller $R L$ than its counterpart, both for losses caused by $\alpha$ and for those caused by finite gratings. In all cases the $R L$ reduces sharply with increasing $K_{c}$, on the other hand larger $K_{c}$ broadens the resonance peak (reduces the mirror $Q$ factor), a fact which might be considered disadvantageous. The configurations of 
Fig. 1 cannot shape the resonance peak and a $-1 /-10 \mathrm{~dB}$ form factor of $\sim 17 \%$ was found to be common.

In Fig. 8 we plotted the resonance peaks for the mirror of Fig. 1a) for two sets of ring mode numbers $N$ and for two $K_{c}$ values, namely 0.02 and 0.05 . The larger $N$ produces narrower, the larger $K_{c}$ produces wider $B W$. The corresponding $B W$ for the other mirror configuration is significantly narrower, and the $B W$ dependence on $N$ considerably reduced from that shown in Fig. 8.

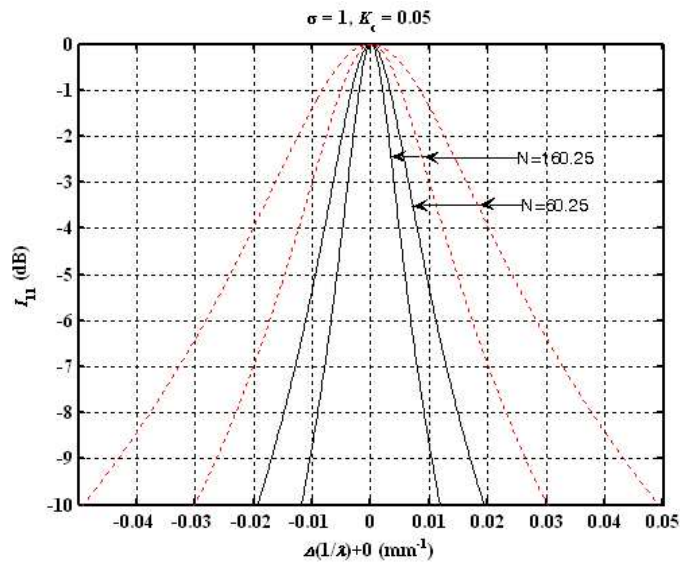

Fig. 8. The central peak of the reflected intensity of the mirror of Fig. 1a). The parameter is the ring mode number, solid lines: $K_{c}=0.02$, dotted lines: $K_{c}=0.05$. Multiply the abscissa by $3 \times 10^{2}$ to obtain the $B W$ in $\mathrm{GHz}$

The mirrors can be tuned by adjusting fractionally one or more mode numbers, i.e., optical lengths by, for example, thermo-optic means. The location of the main resonance is insensitive to $K_{c}$ or to loss. Fractional increase of a mode number reduces the resonance frequency but the rate of change depends on the type of the mirror (Fig.1a, or 1b) as well as on the main mode number $N$. Detailed results will be presented elsewhere; here we must restrict ourselves to stating that the mirror of Fig. 1a) produced a $-12 \mathrm{GHz}$ shift for $\Delta N=+0.01$ at $N=60$, whereas the mirror of Fig. $1 \mathrm{~b}$ ) produced a $-3 \mathrm{GHz}$ shift under the same conditions. The magnitude of these shifts decreases with increasing $N$, but not proportionally. Further investigations of parameter sensitivities for the mirrors are in progress.

\section{CONCLUSION}

Two grating-assisted microring reflectors, suitable as laser mirrors have been analytically and numerically investigated. Selection rules governing the proper mode numbers of the ring and the arms of the Bragg gratings for centering the reflection band were discussed. The effect of loss, both waveguide loss and leakage loss from finite gratings, on the reflected signal, the effect of coupling on bandwidth and the rate of tunability have been documented. Comparisons between the mirrors allow for making a judicious choice.

\section{ACKNOWLEDGEMENT}

The support of the Spanish Ministry of Education project CICYT TEC2006-13273-C03-03/MIC and the Natural Sciences and Engineering Research Council of Canada are acknowledged.

\section{REFERENCES}

[1] I. Chremmos, and N. Uzunoglu, "Reflective properties of double-ring resonator system coupled to a waveguide," IEEE Photon. Technol. Lett., vol. 17, no. 10, pp. 2110-2112, 2005.

[2] Y. Chung, D.-G. Kim, and N. Dagli, "Widely tunable coupledring reflector laser diode," IEEE Photon. Technol. Lett., vol. 17, no. 9, pp. 1773-1775, 2005.

[3] J.K.S. Poon, J. Scheuer, and A. Yariv, "Wavelength-selective reflector based on a circular array of coupled microring resonators," IEEE Photon. Technol. Lett., vol. 16, no. 5, 13311333, 2004.

[4] G.T. Paloczi, J. Scheuer, and A. Yariv, "Compact microringbased wavelength-selective inline optical reflector," IEEE Photon. Technol. Lett., vol. 17, no. 2, pp. 390-392, 2005.

[5] O. Schwelb, "Band-limited optical mirrors based on ring resonators: analysis and design," J. Lightw. Technol., vol. 23, no. 11, pp. 3931-3946, 2005.

[6] B. Liu, A. Shakouri, and J.E. Bowers, "Wide tunable double ring resonator coupled lasers," IEEE Photon. Technol. Lett., vol. 14 , no. 5, pp. 600-602, 2002.

[7] D.B. Mortimore, "Fiber loop reflectors," J. Lightw. Technol., vol. 6, no. 7, pp. 1217-1224, 1988.

[8] C. Vázquez, S. Vargas, and J.M.S. Peña, "Design and tolerance analysis of a router with an amplified resonator and Bragg gratings," Appl. Optics, vol. 39, no. 12, pp. 1934-1940, 2000.

[9] C. Vázquez, J. Montalvo, P.C. Lallana, and J.M.S. Pena, "Applications of recirculating optical configurations on filters and lasers," Microtechnologies for the New Millennium 2005, Proc. SPIE, vol. 5840, pp. 315-324, Sevilla (España) 9-11 May, 2005.

[10] C. Vázquez, S. Vargas, and J.M.S. Pena, "Sagnac loop in Ring Resonators for Tuneable Optical Filters, “ J. Lightw. Technol., vol. 23, no. 8, pp. 2555-2567, 2005.

[11] C. Vázquez, S. Vargas, J.M.S. Pena, and P. Corredera, "Tunable Optical Filters Using Compound Ring Resonators for DWDM," IEEE Photonics Technol. Lett., vol. 15, no. 8, pp. 1085-1087, 2003

[12] O. Schwelb, "Generalized analysis for a class of linear interferometric networks - Part II: simulations," IEEE Trans. Microwave Theory Tech., vol. 46, no. 10, pp. 1409-1418, 1998.

[13] B. Vizoso, C. Vázquez, R. Civera, M. López-Amo, and M.A. Muriel, "Amplified fiber-optic recirculating delay lines," $J$. Lightw. Technol., vol. 12, no. 2, pp. 294-305, 1994.

[14] D.G. Rabus, M. Hamacher, U. Troppenz and H. Heidrich, "High- $Q$ channel-dropping filters using ring resonators with integrated SOAs," IEEE Photonics Technol. Lett., vol. 14, no. 10, pp. 1442-1444, 2002.

[15] O. Schwelb, and I. Frigyes, "Series-coupled microring resonator filters with embedded semiconductor optical amplifiers," Microwave \& Opt. Technol. Lett., vol. 42, no. 5, pp. 427-432, 2004.

[16] H.M. Stoll, "Optimally coupled, GaAs-distributed Bragg reflection lasers," IEEE Trans. Circuits Syst., vol. CAS-26, no. 12, pp. 1065-1072, 1979.

[17] O. Schwelb, "Resonance splitting and its invariance in coupled optical microring resonators," Presented at Photonics North 2007, Ottawa, Canada, May 4-6, 2007. 